\title{
A Methodological Argument Against Scientific Realism
}

\author{
Darrell P. Rowbottom \\ darrellrowbottom@ln.edu.hk
}

First, I identify a methodological thesis associated with scientific realism. This has different variants, but each concerns the reliability of scientific methods in connection with acquiring, or approaching, truth or approximate truth. Second, I show how this thesis bears on what scientists should do when considering new theories that significantly contradict older theories. Third, I explore how vulnerable scientific realism is to a reductio ad absurdum as a result. Finally, I consider which variants of the methodological thesis are the most defensible in light of the earlier findings.

\section{The methodological thesis of scientific realism}

Advocates of scientific realism tend to take the position to involve a methodological claim, which concerns the reliability of the scientific method (or scientific methods), although this is not explicitly stated in many presentations of the position. For example, Psillos (1999, xix) initially characterizes scientific realism in terms of three core theses: metaphysical, semantic, and epistemic. ${ }^{1}$ A couple of pages later, however, he adds:

It should be taken to be implicit in the realist thesis that the ampliative-abductive methods employed by scientists to arrive at their theoretical beliefs are reliable: they tend to generate approximately true beliefs and theories. (Psillos 1999, xxi)

\footnotetext{
${ }^{1}$ The metaphysical thesis is that the world has 'a definite and mind-independent natural kind
} 
Such a thesis is significant because realists do not want to allow that the aforementioned epistemic thesis - 'mature and predictively successful scientific theories are well-confirmed and approximately true of the world' (Psillos 1999, xix) - is true as a matter of mere luck. Similar but significantly distinct statements may be found in the work of other well-known scientific realists. For example, Boyd (1980, 613-614) writes that:

[Progress in science] is achieved by a process of successive approximation: typically, and over time, the operation of the scientific method results in the adoption of theories which provide increasingly accurate accounts of the causal structure of the world.

Let's call these variants of the methodological thesis of scientific realism. This nomenclature is reasonable in so far as the other theses of scientific realism also have different variants. In short, scientific realism is ecumenical. It is 'perhaps only a slight exaggeration to say that scientific realism is characterized differently by every author who discusses it', in the words of Chakravartty (2011). As a result, it is imprudent to think that every scientific realist endorses (a variant of) the methodological thesis. ${ }^{2}$ So I will limit my discussion, in what follows, to forms of scientific realism incorporating such a thesis. My aim is to explore the prospects for such forms of scientific realism, and especially their vulnerability to criticisms of a methodological character.

\footnotetext{
${ }^{2}$ For a detailed analysis of scientific realism with reference to the history of philosophy of science, and an argument that the methodological thesis is central to scientific realism, see Rowbottom (2019a) and Rowbottom (2019b, chapter 3 \& appendix). For different methodological theses pertaining to realism, see Hendry (1996) and Wray (2015).
} 


\section{The methodological argument against scientific realism}

The methodological thesis is methodological not only in so far as involves a claim about what scientific methods (or 'the scientific method') can reliably achieve. It is methodological (in at least some variants) also in so far as it has consequences about the way that science should be done. So if those consequences are false, it follows that the methodological thesis is false. In essence, that's to say, there's a potential reductio of scientific realism (involving a methodological thesis), based on what it says scientists should do. For reasons we will shortly see, this argument is probabilistic in character.

Consider Psillos's variant of the methodological thesis, in the first instance. Let $b$ denote (the propositional content of) a 'theoretical [scientific] belief', AM denote 'was arrived at by ampliative-abductive [scientific] methods', and $\approx$ denote 'is approximately true'. If we construe reliability in terms of probability - as is standard in reliability engineering, where reliability is defined as unity minus probability of failure - then we may take the thesis to have the following form: ${ }^{3}$

$$
\mathrm{P}(\approx b \mid \mathrm{AM} b) \geq n
$$

The intended value of $n$ is difficult to judge from an unqualified reliability claim, such as Psillos's. Nonetheless, it is plausible that it must be considerably greater than one half, considering the social norms governing reliability claims. For example, we would not say that

\footnotetext{
${ }^{3}$ For an introduction to the use of probability in reliability engineering, see Ebeling (1997, chapter 2). The appropriate interpretation of probability will be aleatory: a relative frequency in the limit, a long-run propensity (Gillies 2000), or some such.
} 
perception is a reliable way of forming beliefs were it to transpire that perception-based beliefs are wrong around 30 to 40 percent of the time. However, we should also be wary about lumbering the advocate of the methodological thesis with too high a value for $n$, and hence an implausible view on its face. So I propose to set $n$ at 0.8 , for the purposes of subsequent illustrative calculations. It is easy to see how varying $n$ affects the results, so this is left as an exercise for the reader - and especially for any readers who think that 0.8 lies outside a reasonable interval.

A word about the scope of this methodological thesis is also in order. When precisely is a theoretical belief 'arrived at'? And should we be concerned with the theoretical beliefs of individual scientists, or only with those of groups of scientists (such as the workers, or failing that just the experts, in any given field)? In what follows, I will assume that the thesis only holds for theoretical beliefs on which there is considerable consensus on the part of a community with appropriate expertise (as could be measured, perhaps, by an appropriate belief aggregation function). As will soon become apparent, this is a charitable assumption in context. For if one were instead to hold that ampliative-abductive scientific method(s) are reliable on any given use, by any given scientist, then the argument presented below would be stronger (in so far as theoretical belief changes would be much more numerous).

Now imagine that a scientific community is convinced that $\mathrm{P}(\approx b \mid \mathrm{AM} b) \geq 0.8 .^{4}$ How should this affect their reasoning when faced with a potential change in scientific belief that would

\footnotetext{
4 This assumption is only made for illustrative purposes. The crucial point is that the methodological thesis suggests that scientists should do something; hence by endorsing the thesis, a scientific realist implicitly offers advice to scientists.
} 
strongly contradict old beliefs arrived at by ampliative-abductive scientific methods ${ }^{5}$ Before I answer this question, I must explain the notion of strong contradiction. In general, $p$ strongly contradicts $q$ precisely when $\approx p$ contradicts $\approx q$. The importance of strong contradiction, in the present context, is that it is potentially problematic for scientific realists (committing to the methodological thesis) in a way that weak contradiction - i.e., contradiction that fails to be strong - is not. The classification of pairs of propositions as strongly or weakly contradictory is reasonably straightforward in many cases. For example, 'Clara went to the cinema and took a stroll around the lake today' only weakly contradicts 'Clara went to the cinema and took a stroll along one side of the lake today', whereas each of the aforementioned propositions strongly contradicts 'Clara neither went to the cinema nor took a stroll today'. Similarly, a scientific realist might claim that 'Electrons possess only the intrinsic properties of mass and charge' weakly contradicts 'Electrons possess only the intrinsic properties of mass, charge, and spin', while granting that 'Electrons do not exist' strongly contradicts each of the previous propositions.

Now let $c$ represent the potential new belief being considered by the community, and $\left\{b_{1}, \ldots\right.$, $\left.b_{m}\right\}$ represent the set of strongly contradictory old scientific beliefs selected by ampliativeabductive methods. Ex hypothesi: $\mathrm{P}\left(\approx b_{1} \mid \mathrm{AM} b_{1}\right) \geq 0.8, \ldots, \mathrm{P}\left(\approx b_{\mathrm{m}} \mid \mathrm{AM} b_{m}\right) \geq 0.8$. Thus, from the axioms of probability: $\mathrm{P}\left(\neg \approx b_{1} \mid \mathrm{AM} b_{1}\right) \leq 0.2, \ldots, \mathrm{P}\left(\neg \approx b_{m} \mid \mathrm{AM} b_{m}\right) \leq 0.2$. Provided that each iteration of the process is independent, it also follows that the probability that no member of $\left\{b_{1}, \ldots, b_{m}\right\}$ is approximately true, given the methodological evidence, is as follows:

\footnotetext{
${ }^{5}$ Not all changes in scientific belief are like this. Some new beliefs do not contradict old ones. Moreover, some old beliefs may not be based on scientific methods.
} 
$\mathrm{P}\left(\neg \approx b_{1} \& \ldots \& \neg \approx b_{m} \mid \mathrm{AM} b_{1} \& \ldots \& \mathrm{AM} b_{m}\right) \leq 0.2^{m} . \quad$ Thus, since $\approx c$ entails $\neg \approx b_{1} \& \ldots \& \neg \approx b_{m}$, $\mathrm{P}\left(\approx c \mid \mathrm{AM} b_{1} \& \ldots \& \mathrm{AM} b_{m}\right) \leq 0.2^{m}$. In short, the maximum value of $\mathrm{P}\left(\approx c \mid \mathrm{AM} b_{1} \& \ldots \& \mathrm{AM} b_{m}\right)$ drops sharply as a function of $m$. When $m=2$ it is $1 / 25$, when $m=4$ it is $1 / 625$, when $m=6$ it is $1 / 15625$, when $m=8$ it is $1 / 390625$, and so on. Note also that the relevant set of old scientific beliefs, $\left\{b_{1}, \ldots, b_{m}\right\}$, need not be exhausted by active beliefs at the time the potential new belief, $c$, is proposed. Even discarded beliefs that strongly contradict $c$ prove evidentially relevant to $c$, provided they were formed in the correct way, according to the methodological thesis. Thus some of the advice to scientists from the methodological thesis is as follows: keep a detailed historical record of past theories, classified by domain, and consult the relevant domain record in depth (in its entirety) whenever you consider theory change. The very fact that scientists don't normally follow this advice may be an early cause for realist concern, in so far as we think actual science is done in a good way (i.e. that actual scientific methods for theory selection are good, as realists themselves tend to suggest). But I will say no more about this interesting topic here.

There are several intricacies that require further discussion. For instance, how should beliefs be individuated? And what if some members of $\left\{b_{1}, \ldots, b_{m}\right\}$ are strongly inconsistent with each other? But let's postpone discussion of such issues in order to consider the significance of the result above. For an initial flavor of how this result provides a potential basis for an

\footnotetext{
${ }^{6}$ I will return to the assumption of independence later. For the moment, note merely that it is not necessary. For example, approximate independence will be sufficient for the argument to go through. Note also that assuming some forms of dependence of a stronger character is compatible with - and may even strengthen - some of the results that follow in the main body. For instance, allowing that $\mathrm{P}\left(\approx b_{1} \mid \mathrm{AM} b_{1} \& \mathrm{AM} b_{2}\right)$ is greater than $\mathrm{P}\left(\approx b_{1} \mid \mathrm{AM} b_{1}\right)$, when $b_{1}$ only weakly contradicts $b_{2}$, is unproblematic.
} 


\section{PREPRINT - In Press at Synthese}

argument against the methodological thesis (and hence realism incorporating this), consider the following scenario. A new theory $(c)$ is proposed, which is precisely as good as the dominant theory in the domain $\left(b_{m}\right)$ on the non-methodological measures scientists should use when forming prior probabilities about it (given their background information). So $c$ saves the known phenomena as well as $b_{m}$ (in conjunction with appropriate auxiliary hypotheses and models), and $c$ is also as theoretically virtuous as $b_{m}$ in all other measurable respects (e.g., simplicity, consistency with other contemporary theories in different domains, and potential explanatory power). However, $c$ strongly contradicts $b_{m}$ and all its predecessors, although each was selected via ampliative-abductive scientific methods. Would scientists be correct to refuse to treat $c$ as a serious contender, because of the way it diverged from previous theories in the domain?

A more challenging kind of scenario for the methodological thesis is also easy to envisage. Imagine now that the non-methodological evidence strongly favours the new theory $(c)$. Imagine, for instance, that $c$ is more accurate than any predecessor (when conjoined with appropriate auxiliaries and deployed via appropriate models), is more simple than any predecessor, and has greater scope than any predecessor. Imagine further that $c$ is at least equally as virtuous as any predecessor in other measurable dimensions, such as consistency with other existing theories. According to the methodological thesis, it appears possible nonetheless for the methodological evidence to show that $c$ should not be treated as a serious contender (for truth-likeness).

Bayesian confirmation theory can be used to illustrate this, with the benefit of some simplifying assumptions. Let $B$ represent the background information assumed by the scientists; note that this includes the methodological thesis - for ease, take this to be 
$\mathrm{P}(\approx b \mid \mathrm{AM} b)=0.8$ - which they accept on the testimony of their realist colleagues in philosophy. ${ }^{7}$ And let $e$ represent $\left\{\mathrm{AM} b_{1}, \ldots, \mathrm{AM} b_{m}\right\}$; this is the methodological evidence from the history of science. From Bayes's theorem, we know:

$$
P(\approx c \mid e \cdot B)=\frac{P(\approx c \mid B) P(e \mid \approx c . B)}{P(e \mid B)}
$$

To simplify the illustration, assume the scientists also accept that either $c$ is approximately true or $b_{m}$ is approximately true (on their background information): $\mathrm{P}(\approx c \mid B)+\mathrm{P}\left(\approx b_{m} \mid B\right)=1$. Then we may rewrite the previous equation as follows:

$$
P(\approx c \mid e \cdot B)=\frac{P(\approx c \mid B) P(e \mid \approx c . B)}{P(\approx c \mid B) P(e \mid \approx c . B)+P\left(\approx b_{m} \mid B\right) P\left(e \mid \approx b_{m} \cdot B\right)}
$$

We now need to determine values for the terms on the right hand side. Let's deal with the prior probabilities, $\mathrm{P}(\approx c \mid B)$ and $\mathrm{P}\left(\approx b_{m} \mid B\right)$, first. The former is much higher than the latter in the class of scenario under consideration, because $c$ is more empirically accurate, simple, and so forth, than $b_{m}: \mathrm{P}(\approx c \mid B)>>\mathrm{P}\left(\approx b_{m} \mid B\right)$. So let's imagine $\mathrm{P}(\approx c \mid B)=0.9$ and $\mathrm{P}\left(\approx b_{m} \mid B\right)=0.1$. Now we need only determine the remaining likelihoods.

Unfortunately, this is not as straightforward as it might first appear; and the formal treatment must be complicated accordingly. ${ }^{8}$ To see this, consider the case where $m$ is unity. We seek

\footnotetext{
${ }^{7}$ Although this is a conditional statement about an aleatory probability, it's reasonable to think that the scientists should set their subjective probabilities (or intersubjective probability) to the same value.
} 
values for $\mathrm{P}\left(\mathrm{AM} b_{1} \mid \neg \approx b_{1} . B\right)$ - because $c$ and $b_{1}$ are strongly inconsistent and exhaustive ex hypothesi - and for $\mathrm{P}\left(\mathrm{AM} b_{1} \mid \approx b_{1} . B\right)$. The former ought to be low, for someone who takes scientific methods to reliably select truth-like theories (on each iteration). However, the value of the latter might vary considerably depending on context. For example, one might expect it to be zero if there were uncountably many approximately true theories in the domain of $b_{1}$, or extremely low if said domain were finite but had not been intentionally investigated by scientists. One might expect it to take a much higher value, on the other hand, if there were a small finite number of theories weakly inconsistent with $b_{1}$ and the aforementioned domain had been investigated extensively.

One way out of this impasse is as follows. Let $B$ contain the information that the domain of $b_{m}$ has been actively investigated by scientific methods. Let $w\left(b_{m}\right)$ represent the set of all theories which are weakly inconsistent with $b_{m}$, and let AM now represent the claim that scientific methods select a member of the specified set (or, as previously, the specified item). Advocates of the methodological thesis would expect $\mathrm{P}\left(\mathrm{AM} w\left(b_{1}\right) \mid \approx b_{1} \cdot B\right)$ to be high and $\mathrm{P}\left(\mathrm{AM} w\left(b_{1}\right) \mid \neg \approx b_{1} . B\right)$ to be low (in line with values for $\mathrm{P}\left(\mathrm{AM} b_{1} \mid \neg \approx b_{1} . B\right)$ ). That is, provided that the overlap between $w\left(b_{1}\right)$ and $w(t)$ is taken to be considerable, when $t$ represents the true theory in the domain, as it presumably should be. In short, the underlying notion is as follows. Pick two theories at random from $w(t)$. The probability that they weakly contradict each other is much higher than the probability that they strongly contradict each other. Thus

${ }^{8}$ It is also possible to construct a Bayesian argument by appeal to $\mathrm{P}(\approx b \mid \mathrm{AM} b)$ and $\mathrm{P}(\neg \approx b \mid \mathrm{AM} b)$ more directly. This shows that if one elects to believe $c$, in scenarios of the kind under consideration, then one should lower one's confidence in the methodological thesis (and often disbelieve in it) as a result. This appears to be more closely related to the argument from unconceived alternatives (Stanford 2006), however, so I do not cover it here. 
provided there's a high chance of selecting two theories from $w(t)$ in two applications of the relevant scientific method(s), there's also high chance of selecting two theories that weakly contradict one another. For the remainder of this section, in the interests of simplicity, I will treat the two chances as if they're the same; that's to say, the mathematical treatment will be such that $w\left(b_{1}\right)$ and $w(t)$ are co-extensive. I will return to this issue in the next section.

So let's assume $\mathrm{P}(\mathrm{AM} w(b) \mid \approx b . B)=0.8$ and $\mathrm{P}(\mathrm{AM} w(b) \mid \neg \approx b . B)=0.2$, and that the results on any application of ampliative-abductive methods are independent. And let $e$ now be $\left\{\mathrm{AM} w\left(b_{1}\right)\right.$, $\left.\ldots, \mathrm{AM} w\left(b_{m}\right)\right\}$, which is entailed by what we previously took to be $e$ (and might be understood to be the only evidentially significant content therein in this context). Now $\mathrm{P}(e \mid \approx c . B)$ is $0.2^{m}$, because all members of $\left\{b_{1}, \ldots, b_{m}\right\}$ are strongly inconsistent with $c$. $\mathrm{P}\left(e \mid \approx b_{m} . B\right)$ will vary depending on how many members of $\left\{b_{1}, \ldots, b_{m}\right\}$ strongly contradict, rather than weakly contradict, $b_{m}: \mathrm{P}\left(e \mid \approx b_{m} . B\right)=0.2^{s} 0.8^{m-s}$, where $s$ represents the number of members of the set that strongly contradict $b_{m}$. We have the following:

$$
P(\approx c \mid e . B)=\frac{0.9 * 0.2^{m}}{\left(0.9 * 0.2^{m}\right)+\left(0.1 * 0.2^{s} * 0.8^{m-s}\right)}
$$

We are now in a position to plug some numbers in. Even if $m$ is as low as $4, \mathrm{P}(\approx c \mid e . B)=0.034$ provided that $s$ is zero. Raising $m$ slightly to 6 and letting $s$ be 1 gives a notably lower value: $\mathrm{P}(\approx c \mid e . B)=0.0087$. In either instance, $c$ appears to be a theory that is barely worth any serious consideration as a candidate truth-like theory. For example, if we allow that the division of theoretical labor in a scientific domain should be in proportion to the probability of the theoretical alternatives on the evidence, then a very small fraction of labor in the relevant domain should be on $c$ rather than $b_{m}$. Yet this appears wrong on its face, and the rule about the division of theoretical labor does not appear to be the culprit (in so far as one could 
imagine similar results arising even if the rule were relaxed somewhat). ${ }^{9}$ It appears to be wrong to think that the application of ampliative-abductive methods can provide us with such strong evidence.

Before I close this section, I should like to make two further points concerning the argument above. First, Psillos's version of the methodological thesis concerns all scientific beliefs of a theoretical variety. It does not only concern scientific theories. So one might instead consider how beliefs about what populates the unobservable realm vary over time in response to repeated use of scientific methods. Prima facie, this tends to make the argument look stronger, in so far as beliefs about what exists may change independently of other theoretical beliefs. That's to say, one might think that a token of a posited unobservable kind has been detected - a Higgs boson or a gravitational wave, for instance - without this changing one's theoretical beliefs (although it might strengthen one's confidence in some such beliefs).

${ }^{9}$ Naturally, realists may allow that $c$ could be preferred for practical purposes, e.g. as a calculation device. But they would think of it only as a temporary fix. Realists may also allow that $c$ would serve as an excellent heuristic for future inquiry in the domain. For example, they might suggest that a search should be conducted for theories preserving $c$ 's structure (or much of its structure) but with ontologies similar to past theories. However, the reductio relies on the idea that it would be implausible that $c$ was merely a temporary fix or a heuristic. Its discovery appears to constitute (cognitive) scientific progress, rather than a means by which to make such progress. Acceptance of this is at odds with typical realist views of (cognitive) scientific progress, such as those due to Bird (2007) and Niiniluoto (2017), on which increases of theoretical truth-likeness are necessary for progress to occur. It would be possible for a realist to sacrifice such a view on progress to resist the argument, but I will assume herein that they'd prefer to resist it in another way. 
Second, the thesis doesn't say anything about how to individuate beliefs, and therefore holds, as it stands, for highly complex beliefs as well as simple beliefs. But this has an interesting consequence. Take simple beliefs to have atomic propositions (i.e., propositions which cannot be broken down into other propositions) as their objects, and complex beliefs to have non-atomic (or complex) propositions as their objects. Moreover, take the complexity of a proposition to be a function of the number of atomic propositions that it decomposes into. Then as complexity rises, we would expect probability to decrease for complex propositions that are conjunctions of atomic propositions. That's because $\mathrm{P}(p \& q) \leq \mathrm{P}(p)$ follows from the axioms of probability, and very often, when $p$ and $q$ are empirical claims, it is evident that $\mathrm{P}(p \& q)<\mathrm{P}(p)$. Consider, for example, 'Fido is an animal' and 'Fido has eyes', or 'There is at least one atom' and 'Atoms have nuclei'. Now here's the interesting consequence. Take any extremely complex conjunctive scientific belief arrived at by ampliative-abductive methods. According to the methodological thesis, this complex belief was formed by reliable means, so should probably be true. But this means that many of the atomic beliefs of which it is formed must have considerably higher probabilities still; to see this, think about stripping out one atomic proposition at a time and comparing the probability of this with the remainder. And often many of those atomic propositions will themselves have been arrived at by ampliativeabductive methods. The upshot of this may very well be that the reliability of ampliativeabductive methods is a function of the complexity of their (conjunctive) outputs on the methodological thesis. But this suggests that sometimes - for instance, when considering atomic existential claims - those methods must be even more reliable than assumed above.

In the remainder of this paper, I will turn my attention to how realists might respond to the challenge developed in this section. For brevity, I will refer to it as 'the argument'. 


\section{Possible responses to the argument}

From a realist perspective, two kinds of response to the argument are available. First, one might argue that there are problematic assumptions - either explicit, via stipulation, or implicit, via omission - in the argument. Second, one might argue that an alternative version of the methodological thesis is preferable. The routes are not mutually exclusive, and taking either is compatible with biting the bullet to some extent.

In the remainder of the paper, I will consider each avenue in turn. I will use sub-headings for ease of reference. Before I turn to this, however, let me stave off one concern that might arise, which is that the methodological argument is old wine in a new - more jazzy, more technical - bottle. To be more specific, isn't the methodological argument simply a repackaging of the pessimistic meta-induction, the argument from unconceived alternatives (Stanford 2006), or some more generalized underdetermination-related - Duhemian or Quinean - concerns? The easy way to see that the answer lies in the negative is as follows. I have not made any kind of inductive move about future science; I have not suggested that future theories will typically have central terms that don't refer, or that serious unconceived alternative theories will typically be available. Moreover, recall that the direct target of the methodological argument is a particular kind of methodological thesis associated with scientific realism. It only indirectly bears on the other theses, such as the epistemic thesis. ${ }^{10}$

\footnotetext{
${ }^{10}$ The methodological argument is also distinct from the claim advanced by Stanford (2015, 873-874) that:
} 
Independence \& the correspondence principle

$[\mathrm{T}]$ he classical scientific realist can afford to be cavalier or even enthusiastic about evidence of increasing theoretical conservatism in science. After all, she thinks that contemporary theories have things sorted out at least roughly right and that our remaining errors are simply errors of detail. She is confident that the theories embraced by future scientific communities will seem both to us and to the members of those communities simply to be corrected, expanded, and more sophisticated versions of the ones that we ourselves have accepted.

Stanford here focuses on how belief in the approximate truth of contemporary theories primarily, in the epistemic component of scientific realism - influences expectations about how science will change (and attitudes towards some kinds of change). He does not consider the methodological thesis directly (although he does, in the course of his discussion, consider how changes in a community might affect the extent to which it is vulnerable to the problem of unconceived alternatives). Thus he doesn't consider how the historical record of theories selected by appropriate methods might be evidentially significant on some variants of scientific realism, and exactly how said record might be relevant. Recall, for example, my discussion of how advocates of Psillos's variant of the methodological thesis might recommend scientists to keep better historical records than they do, and to carefully consult such records when considering theory change. (I also prefer not to use the category of 'classical scientific realists', because philosophers who would typically be identified as falling into that camp, such as Boyd and Psillos, endorse different variants of the methodological thesis.) 
At several junctures in the previous section, independence was assumed. But what if the result of an application of ampliative-abductive scientific methods (in some domain) depends on the results of the prior application(s) of those methods? For example, is it correct that $\mathrm{P}\left(\mathrm{AM} b_{1} \& \mathrm{AM} b_{2}\right)=\mathrm{P}\left(\mathrm{AM} b_{1}\right) \mathrm{P}\left(\mathrm{AM} b_{2}\right)$ in a typical case Or should one instead recognize that normally $\mathrm{P}\left(\mathrm{AM} b_{1} \& \mathrm{AM} b_{2}\right)=\mathrm{P}\left(\mathrm{AM} b_{1}\right) \mathrm{P}\left(\mathrm{AM} b_{2} \mid \mathrm{AM} b_{1}\right) ?^{11}$

As mentioned in footnote six, the argument only relies on the fact that independence holds to enough of an extent to make methodological evidence have significant force (when values of $m$ remain reasonably small). So this means it is compatible with accepting dependence, and even accepting that $\mathrm{P}\left(\mathrm{AM} b_{2} \mid \mathrm{AM} b_{1}\right)>\mathrm{P}\left(\mathrm{AM} b_{2}\right)$ - to stick with the same simple example. The extent to which its force is diminished depends on exactly how much greater one takes the former to be than the latter. It is only seriously threatened if $\mathrm{P}\left(\mathrm{AM} b_{2} \mid \mathrm{AM} b_{1}\right)>>\mathrm{P}\left(\mathrm{AM} b_{2}\right)$, or if some appropriately similar principle, such as $\mathrm{P}(\mathrm{AM} w(b) \mid \mathrm{AM} b)>>\mathrm{P}(\mathrm{AM} w(b))$ typically holds.

Assuming dependence (even of such a strong variety) is also potentially double-edged, in so far as dependence of future generations of theories on older generations might serve to entrench errors, as well as to preserve accurate (or truth-like) elements thereof. Moreover, methodological principles concerning how new theories should relate to their predecessors do not typically suggest that ontological continuity is required. Consider, for example, Post's (1971, 228) proposal:

\footnotetext{
${ }^{11}$ Unconditional probabilities are used here for presentational purposes. One may imagine that each of these probabilities is conditional on the same information, such as the approximate truth of the beliefs they refer to, if desired. I shall return to this below.
} 


\section{PREPRINT - In Press at Synthese}

The most important heuristic restriction is the General Correspondence Principle. Roughly speaking, this is the requirement that any acceptable new theory L should account for the success of its predecessor S by 'degenerating' into that theory under those conditions under which $\mathrm{S}$ has been well confirmed by tests.

Ensuring that $b_{2}$ degenerates into $b_{1}$ in such contexts may entail - or at least significantly raise the probability of - preserving structure. But it is dubious that two inconsistent theories with similar structures are significantly more likely to be weakly inconsistent with one another than two inconsistent theories that lack similar structures. Compare Bohm's theory of quantum mechanics and the Orthodox theory, for instance. The former posits a deterministic situation, with fundamental particles having definite positions, whereas the latter does not. Moreover, the latter says that spin is an intrinsic property of such particles, whereas the former does not. (Some variants of Bohm's theory also say there is a quantum potential and that the wavefunction exists in multiple dimensions. The Orthodox theory denies these claims.) The two theories appear to be strongly inconsistent as a result of these differences, despite the similarity of their mathematical structures.

Appeal to the partial overlap of $w(t)$ and $w(b)$ when $b \in w(t)$

Assume the selection process only concerns theories. As noted in the previous section, the probability that $b_{1}$ and $b_{2}$ only weakly contradict one another, given that they are members of $w(t)$, is less than unity even if it is high.

An interesting consequence is that even if scientists were guaranteed to select members of $w(t)$ on each application of their ampliative-abductive methods, they might nevertheless 
select some strongly contradictory theories. In fact, the chance of picking (at least) two strongly contradictory theories would increase markedly as the number of (random) picks from $w(t)$ increased. Imagine, for instance, that the probability that any two theories in $w(t)$ strongly contradict one another were 0.1 . Then the probability of the presence of strong contradiction in an $n$-sized randomly selected set of theories in $w(t)$ would be $1-0.9^{3}=0.271$ when $n=3,1-0.9^{6}=0.47$ when $n=4,1-0.9^{10}=0.65$ when $n=5$, and so forth. So it is extremely unlikely that a large set of theories previously selected by scientific methods would exhibit no strong internal contradictions.

However, selecting a new theory that strongly contradicts all other past theories (or even simply the vast majority thereof) remains extremely improbable on such a picture. Indeed, the probability of selecting a new theory that strongly contradicts all other theories in a $n$-sized set of theories which are elements of $w(t)$ would be $0.1^{n}$. Allowing for only partial overlap between $w(t)$ and $w(b)$ when $b \in w(t)$ only serves to diminish the force of the methodological argument somewhat, by slightly reducing the power of the methodological evidence from the history of science. To see this, let's relax the assumption that any application of scientific method(s) is guaranteed to select a member of $w(t)$. Let's assume, instead, that such an application only has a 0.8 probability of selecting a member of $w(t)$ (assuming appropriate background information, which I won't discuss again here). Let's add the false but illustratively useful assumption, which favours the advocate of the methodological thesis in the subsequent calculation, that all elements of $w(t)$ strongly contradict theories that are not elements of $w(t)$. Then the probability of selecting a new theory (by scientific methods) that strongly contradicts all the old $(n)$ theories selected by scientific methods is as follows: $\left(0.8^{*} 0.1+0.2\right)^{n}=0.208^{n}$, as compared to $0.2^{n}$, ex hypothesi, in the argument presented in the previous section. 
Appeal to the history of science

A realist might instead argue that scenarios such as those featuring in the argument rarely occur in science, if at all, and therefore that they are relatively insignificant. For example, $m$ may typically be low. Or other kinds of evidence might typically accrue, over time, which render the past methodological evidence less significant.

In so far as this would be to push the debate into the history of science, which is already a battleground for realists and their opponents, it is not possible to examine whether it's a convincing response in the present paper. (Cherry-picking examples is a ubiquitous risk, and making many random selections from a large sample of cases is the most respectable way to avoid this.) However, the argument has force even if the historical point is conceded. Its crux is as follows: the methodological thesis suggests that scientists should do something unwise in a class of scenarios that are possible in science (even if they are rare).

Improving methods

Another interesting idea is that (ampliative-abductive) scientific methods tend to improve over time: that they tend to become more reliable over time, although they are reliable all of the time. Does this help to refute the argument? For simplicity's sake, we can model this by taking the probability of scientific methods issuing in approximately true claims to increase each time they are used. Imagine, for instance, that $\mathrm{P}\left(\mathrm{AM}_{1} w(b) \mid \approx b . B\right)=0.7$, $\mathrm{P}\left(\mathrm{AM}_{2} w(b) \mid \approx b . B\right)=0.75, \mathrm{P}\left(\mathrm{AM}_{3} w(b) \mid \approx b . B\right)=0.8, \mathrm{P}\left(\mathrm{AM}_{4} w(b) \mid \approx b . B\right)=0.825$, and so forth, where $\mathrm{AM}_{n}$ represents 'is selected by ampliative-abductive methods on use $n$ in the domain'. 
This does have the virtue of lowering the significance of the methodological evidence in the early stages of inquiry relative to the significance of later evidence. But because it still allows that each use of the methods is reliable, it does not help to lessen the force of the methodological argument significantly. Moreover, it has the undesirable consequence (from a realist perspective) of strengthening the force of the methodological evidence when $m$ is high. (The effect of abandoning the claim that methods are always reliable, while maintaining that they improve over time, is examined in the next section.)

Restricting scope

Might the methodological thesis instead be modified in order to render it more defensible? One clear way to do so would be to narrow its scope. Realists have done something similar when devising variants of the epistemic thesis of scientific realism, by introducing qualifiers. Key qualifiers are 'contemporary', 'mature', and 'typically' (or a surrogate such as 'usually' or 'normally'). Let's begin by considering how each of these might be employed.

First, the methodological thesis might be said to concern ampliative-abductive methods only in mature - or even only in contemporary - science. Yet neither approach is effective against the argument. Limiting the methodological thesis to contemporary science - and only contemporary science - is undesirable in so far as it is difficult to see what sort of evidence we could have that it is true now, yet was not beforehand. More significantly, moreover, it is difficult to point to concrete ways in which ampliative-abductive methods in contemporary science differ significantly from those used in historical science (at least over roughly the past century or so). Using 'mature' is more moderate and plausible. It suffices for altering the 
historical battleground, discussed in a previous sub-section, somewhat; the realist need not then be concerned with any ampliative-abductive methods used to posit vital heat or multiple epicycles of motion in astronomy, for instance. However, appeal to maturity does not serve to do much more than this. It remains the case that there are possible scenarios in science albeit now only in mature science - where methodological evidence appears to have too much weight.

Second, more interestingly, scientific methods might be said only to be typically reliable. In effect, this is to make a frequency claim - and hence, plausibly, an aleatory probability claim - at the meta-level. It involves, for instance, setting a high value for $\mathrm{P}(\mathrm{P}(\approx b \mid \mathrm{AM} b) \geq 0.8)$ or $\mathrm{P}(\mathrm{P}(\mathrm{AM} w(b) \mid \approx b . B) \geq 0.8)$. Thankfully, a technical treatment of this modification - which proves to be rather a messy business - is not necessary to appreciate that it serves to lessen the force of the methodological evidence somewhat, although not a great deal. In effect, it introduces some doubt about the veracity of the methodological evidence. The higher $m$ becomes, the less significant this doubt will be overall. For example, the probability of none of the methods being reliable will decrease rapidly. And if one adds the assumption that unreliable methods in science are no worse than chance selections, as realists may wish to, then this further lowers the significance as $m$ increases.

Another way to restrict the scope of the methodological thesis would be to have it concern only some kinds of scientific beliefs (or products). For instance, it could be restricted to theories or to existential claims. Any such move is liable to increase its plausibility somewhat. But it will leave the argument intact for the kinds of scientific beliefs that the restricted methodological thesis pertains to. 


\section{PREPRINT - In Press at Synthese}

\section{Replacing approximate truth}

A bolder strategy, which appears more promising, is to find an appropriate surrogate for approximate truth. In effect, this is what Boyd $(1980,614)$ does, in his variant of the methodological thesis, by suggesting only that successive generations of theories selected by scientific methods typically provide 'increasingly accurate accounts of the causal structure of the world'. And even imagining that $t^{*}$ must be more truth-like than $t$, in the event that $t^{*}$ provides a more accurate account of the causal structure of the world than $t$, nothing about the approximate truth of $t^{*}$ (or $t$ ) follows. Indeed, a theory may be strongly inconsistent with all its predecessors and still provide a more accurate account of the causal structure of the world than any of those predecessors. Thus the argument in section two does not apply to this view. $^{12}$

Because Boyd's talk of 'causal structure' is somewhat difficult to unpack, however, let's consider a simpler alternative with a similar character: a theory selected by scientific ampliative-abductive methods will very probably be closer to the truth than its predecessor. Call this the incremental methodological thesis. Grant also that this is closely associated with other theses - equivalents to those discussed in the argument presented against Psillos's variant of the methodological thesis - such as 'The probability that a theory was selected by

${ }^{12}$ It may be possible to construct a subtly different methodological argument against Boyd's view, however, by considering a scenario in which a new theory is proposed which deviates from prior theories considerably in what it says about the causal structure of the world. One obstacle is that the talk of 'causal structure' is rather vague. And a key worry is that preservation of this structure is compatible with significant ontological change (and is thus more suggestive of structural, rather than scientific, realism). 
scientific ampliative-abductive methods, given that it is less close to the truth than its predecessor, is very low'. How susceptible is this to a something akin to 'the argument'?

Prima facie, the answer appears to be 'not very'. Consider two theories, $t$ and $t^{*}$, such that the latter is closer to the truth than the former. Evidently, $t^{*}$ might be strongly inconsistent with $t$. Moreover, $t^{*}$ and $t$ might share no content whatsoever. Thus, spotting differences in content between new and old theories would not straightforwardly provide grounds for doubting the incremental methodological thesis, or indeed for resisting adoption of a new theory that was strongly inconsistent with - or even shared minimal ontological content with - its predecessors (selected by the relevant methods).

The incremental version of the methodological thesis might also potentially be linked with, or replaced with, another more moderate view: a theory selected by scientific ampliativeabductive methods will probably 'latch onto unobservable reality' (and perhaps latch better onto unobservable reality than its predecessors). The talk of 'latching' is borrowed from Saatsi $(2019,623)$, who explains it as follows:

$T$ LATCHES ONTO UNOBSERVABLE REALITY if and only if $T$ 's degree of empirical adequacy is accounted for by $T$ providing a veridical representation of some aspects of unobservable reality ...

$T^{\prime}$ LATCHES BETTER ONTO UNOBSERVABLE REALITY than $T$ if and only if $T^{\prime}$ is more empirically adequate than $T$, and the boost in empirical adequacy is accounted for by a difference in the respective provisions of veridical representations. 


\section{PREPRINT - In Press at Synthese}

Some realists might object to this account, as it stands, on relatively minor technicalities. Here are two examples of such. First, the definition of latching is compatible with $T$ having a zero degree of empirical adequacy (accounted for by a veridical representation of some aspects of the unobervable world). But this is surely a mere oversight, and is addressed by inserting 'positive' - or perhaps even 'high' - before 'degree of empirical adequacy'. Second, latching better should perhaps be characterised in conditional, rather than biconditional, form. One reason is that two theories may be as accurate in their respective domains - have the same degrees of empirical adequacy in those domains - while having different scope. ${ }^{13}$

The idea behind the latching account is nonetheless clear from the following passage:

Never mind what 'proportion' or 'part' of $T$ 's representational content is involved in accounting for its empirical adequacy.... [T]he notion of latching (better) onto reality is defined in broad terms, to capture all (and only) the gist of the no-miracles intuition. In particular, it is meant to be compatible with various forms of scientific

\footnotetext{
${ }^{13}$ Perhaps Saatsi intends 'empirical adequacy' to be a function of accuracy and scope. He is not explicit about this. Confusion might arise because such an understanding would differ from that provided by van Fraassen $(1980,12)$, which is often assumed in the realism debate:
}

a theory is empirically adequate exactly if what it says about the observable things and events in the world is true- exactly if it 'saves the phenomena.'

In short, a theory may be true in 'what it says about the observable things and events in the world' without saying anything about all, or even most or many, such things and events. 
realism that are all in the business of accounting (in realist terms) for the empirical success of false past theories from our current perspective. (Saatsi 2009, 623-624)

So one reasonable way to understand latching is as involving elements of truth in the theory. And a variant of the methodological thesis construing latching in such as way might claim, for instance, only that a theory requires a significant element of truth in order to be selected by scientific methods. It doesn't follow that any two empirically successful theories will share many, or even any, of the same elements.

\section{Conclusion}

In the first section, I presented a key variant of the methodological thesis of scientific realism, which is advocated by Psillos. In the second section, I showed that there is a strong argument against this methodological view. In the third, I surveyed several different ways of responding to this argument. I found that the best way to resist the argument is to avoid appealing to approximate truth (or some near surrogate) directly, in postulating a methodological thesis (or variants thereof).

\section{Acknowledgements}

This paper is based on talks given at Cambridge University's Philosophy of Science Seminar and Durham University's Institute of Advanced Study. I am grateful to various audience members for comments, and particularly Robin Hendry, Milena Ivanova, Brian Pitts, and Jacob Stegenga. I am greatly indebted to Simon Goldstein and Jiji Zhang for insightful comments on drafts of the paper. 


\section{References}

Bird, A. 2007. 'What Is Scientific Progress?', Noûs 41, 64-89.

Boyd, R. 1980. 'Scientific Realism and Naturalistic Epistemology', PSA 1980 Vol. II, 613662.

Chakravartty, A. 2011. 'Scientific Realism', in E. N. Zalta (ed.), Stanford Encyclopedia of Philosophy.

Ebeling, C. E. 1997. An Introduction to Reliability and Maintainability Engineering. Boston, MA: McGraw-Hill.

Gillies, D. 2000. Philosophical Theories of Probability. London: Routledge.

Hendry, R. F. 1996. 'Realism, History and the Quantum Theory: Philosophical and Historical Arguments for Realism as a Methodological Thesis.' PhD thesis, LSE. URL:

http://etheses.1se.ac.uk/1442/

Laudan, L. 1981. 'A Confutation of Convergent Realism', Philosophy of Science 48, 19-49.

Niiniluoto, I. 2017. 'Optimistic Realism about Scientific Progress', Synthese 194, 39213309.

Post, H. 1971. 'Correspondence, Invariance and Heuristics: In Praise of Conservative Induction', Studies in History and Philosophy of Science 2: 213-255.

Psillos, S. 1999. Scientific Realism: How Science Tracks Truth. London: Routledge.

Rowbottom, D. P. 2019a. 'Scientific Realism: What It Is, the Contemporary Debate, and New Directions', Synthese 196, 451-484.

Rowbottom, D. P. 2019b. The Instrument of Science: Scientific Anti-Realism Revitalised. New York: Routledge.

Saatsi, J. 2019. 'What Is Theoretical Progress of Science?', Synthese 196: 611-631. 


\section{PREPRINT - In Press at Synthese}

Stanford, P. K. 2006. Exceeding our Grasp: Science, History, and the Problem of Unconceived Alternatives. Oxford: Oxford University Press.

Stanford, P. K. 2015. 'Catastrophism, Uniformitarianism, and a Scientific Realism Debate That Makes a Difference', Philosophy of Science 82, 867-878.

van Fraassen, B. C. 1980. The Scientific Image. Oxford: Oxford University Press.

Wray, K. B. 2015. 'The Methodological Defense of Realism Scrutinized', Studies in History and Philosophy of Science 54, 74-79. 